$y_{1}, x y_{1}, \cdots, x^{r-1} y_{1}$ are all solutions while $x^{r} y_{1}$ is not a solution. If $r$ is greater than unity, the solution is said to be repeated. If $y_{1}$ is a repeated solution, then it must also satisfy the equation

$$
n a_{0} D^{n-1} y+(n-1) a_{1} D^{n-2} y+\cdots+a_{n-1} y=0,
$$

that is, the equation obtained from (1) by formal differentiation with respect to $D$. The first elements of the theory of repeated solutions of (1) and a certain more general class of equations thus suggested is developed on a simple postulational basis.

ARNoLd DRESDEN, Secretary of the Section.

\title{
ELEMENTARY INEQUALITIES FOR THE ROOTS OF AN ALGEBRAIC EQUATION.
}

\author{
BY PROFESSOR R. D. CARMICHAEL.
}

(Read before the American Mathematical Society, October 27, 1917.)

1. LET us write the general algebraic equation in each of the following forms:*

$$
\begin{aligned}
& x^{n}=a_{1} x^{n-1}+a_{2}{ }^{2} x^{n-2}+a_{3}^{3} x^{n-3}+\cdots+a_{n}{ }^{n}, \\
& x^{n}=c_{n 1} \alpha_{1} x^{n-1}+c_{n 2} \alpha_{2}^{2} x^{n-2}+\cdots+c_{n n} \alpha_{n}{ }^{n}, \\
& x^{n}=\beta_{1} x^{n-1}+\beta_{2} x^{n-2}+\cdots+\beta_{n},
\end{aligned}
$$

where

$$
a_{i}{ }^{i}=c_{n i} \alpha_{i}{ }^{i}=\beta_{i} \quad(i=1,2, \cdots, n),
$$

and $c_{n 1}, c_{n 2}, \cdots, c_{n n}$ denote the binomial coefficients for the power $n$.

If we let $X$ denote the greatest absolute value of a root of equation (1) and let $\alpha$ denote the greatest absolute value of the quantities $\left|\alpha_{1}\right|,\left|\alpha_{2}\right|, \cdots,\left|\alpha_{n}\right|$, then, as was shown by Carmichael and Mason, $\dagger$ we have $X \geqq \alpha$, the equality sign

* The fruitful and convenient notation employed in the first equation was suggested to me by my friend and colleague, Dr. A. J. Kempner.

$\dagger$ This Bulletin, vol. 21 (1914), pp. 14-22. Carmichael and Mason stated the theorem for the equation whose roots are the reciprocals of those of $(1)$. 
holding in a special case, namely, for the equation $(x-\alpha)^{n}=0$. Birkhoff, in the same volume,* reproduced Carmichael and Mason's proof of this theorem and obtained further an upper bound for $X$, so that on combining the two theorems one has

$$
\alpha \leqq X \leqq \frac{\alpha}{2^{1 / n}-1} .
$$

The theorem in (2) was also proved independently by Jensen. $\dagger$

Notwithstanding the fact that the upper bound in (2) is attained in a special case, namely, that of the equation $2 x^{n}-(x+\alpha)^{n}=0$, it is far greater than needful in the case of certain types of equation, as, for instance, the binomial equation $x^{n}=\alpha^{n}$. Here the roots are in absolute value equal to $\alpha$, while the Birkhoff-Jensen upper bound increases indefinitely as $n$ increases. See an extension of the BirkhoffJensen result in $\S 6$ below.

The upper bound in (2), as well as several related results, was obtained by Fujiwara $\ddagger$ by an ingenious method which we shall employ below in the derivation of several theorems.

2. Carmichael and Mason (loc. cit., pages 21-22) also gave an upper bound to the roots of (1), showing indeed that

$$
X \leqq\left\{1+\left|\beta_{1}\right|^{2}+\left|\beta_{2}\right|^{2}+\cdots+\left|\beta_{n}\right|^{2}\right\}^{\frac{1}{2}} .
$$

Probably this bound is never attained. But if we denote by $B$ the second member of (3) and choose $\epsilon$ any positive quantity, however small, then we can find equations all of whose roots are in absolute value between $B(1-\epsilon)$ and $B$. In fact it is sufficient to take a binomial equation $x^{n}=\beta_{n}$ and choose $\beta_{n}$ sufficiently large. Thus the upper bound in (3) is relatively close, in certain cases at least, when the upper bound in (2) is too great by as large a factor as one pleases. On the other hand, the bound in (3) is unsatisfactory in that it is greater than unity however small the coefficients of the equation may be. Fujiwara§ has employed (3) itself to obtain another bound not having this disadvantage, namely, that given in the relation

(4) $X \leqq\left\{\frac{n^{n}}{(n-1)^{n}=1}\left(\left|\beta_{1}\right|^{2}+\left|\beta_{2}\right|^{2}+\cdots+\left|\beta_{n}\right|^{2}\right)\right\}^{\frac{1}{2} n}$,

* Ibid., vol. 21 (1915), pp. 494-495.

$\dagger$ Nyt Tidsskrift, vol. $26 A$ (1915), pp. 6-13. Some other related results are also given by Jensen.

$\ddagger$ Tôhoku Math. Journal, vol. 10 (1916), pp. 167-171.

$\S$ Tôhoku Math. Journal, vol. 8 (1915), pp. 82-85. 
this being valid provided that the condition

$$
\left|\beta_{1}\right|^{2}+\left|\beta_{2}\right|^{2}+\cdots+\left|\beta_{n}\right|^{2}<\frac{1}{n-1}
$$

is satisfied. He (loc. cit.) has also generalized (3) by showing that, when the roots $x_{1}, x_{2}, \cdots, x_{n}$ of (1) are arranged so that $\left|x_{1}\right| \leqq\left|x_{2}\right| \leqq \cdots \leqq\left|x_{n}\right|$, we have

(6) $\left|x_{p}\right| \leqq\left|x_{1} x_{2} \cdots x_{p}\right|^{1 / p} \leqq\left\{1+\left|\beta_{1}\right|^{2}+\left|\beta_{2}\right|^{2}+\cdots+\left|\beta_{n}\right|^{2}\right\}^{\frac{1}{2} p}$.

Kuniyeda* has given interesting generalizations of (3) and (4), showing that

(7) $X \leqq\left\{1+\left(\left|\beta_{1}\right|^{1+p}+\left|\beta_{2}\right|^{1+p}+\cdots+\left|\beta_{n}\right|^{1+p}\right)^{1 / p}\right\}^{p /(1+p)}$,

(8) $X \leqq\left\{\frac{n^{n}}{(n-1)^{n-1}}\left(\left|\beta_{1}\right|^{1+p}+\left|\beta_{2}\right|^{1+p}\right.\right.$

$$
\left.\left.+\cdots+\left|\beta_{n}\right|^{1+p}\right)^{1 / p}\right\}^{p / n(1+p)},
$$

where $p$ denotes any positive number, the latter relation being certainly valid only when

$$
\left(\left|\beta_{1}\right|^{1+p}+\cdots+\left|\beta_{n}\right|^{1+p}\right)^{1 / p} \leqq \frac{1}{n-1} .
$$

Putting $p=1$ in (7) and (8), we have the special cases (3) and (4).

The theorem in (3) and certain immediate related results have also been given by S. B. Kelleher $\dagger$ (without reference).

For certain other discussions of inequalities related to those given above reference may be made to papers by Kakeya $\ddagger$ and Kojima.§ A new proof of some of the results in the latter paper will be given in the sequel.

3. The principal object of the present paper is to derive (in $\$ \$ 5-10$ ) numerous elementary inequalities for the greatest $\uparrow$ absolute value $X$ of a root of equation (1). In every case the

* Ibid., vol. 9 (1916), pp. 167-173; vol. 10 (1916), pp. 187-188.

$\dagger$ Journ. de Math. (7), vol. 12 (1916), pp. 168-171.

† Tôhoku Math. Journal, vol. 2 (1912), p. 140; vol. 3 (1913), p. 23.

Ibid., vol. 5 (1914), p. 54; vol. 11 (1917), p. 119.

If For two other very interesting inequalities on the roots of algebraic equations see Fejér, Math. Annalen, vol. 65 (1908), pp. 413-423 and Lévy, Nouvelles Annales de Math. (3), vol. 11 (1892), pp. 147-148.

T In an obvious manner these yield corresponding inequalities for the least absolute value of a root of equation (1). 
method is altogether elementary. The results generalize a number of the known inequalities and contain several new ones of interest. It is clear that numerous other inequalities may be obtained by a further use of the elementary methods here employed.

4. The formula given by Carmichael and Mason (loc. cit., pages 21-22) for the exact value of $X$ may be put into a shape more convenient for certain purposes than the determinant form in which they presented it. Let us consider the equation

$$
1-a_{\mathrm{i}} z-a_{2}{ }^{2} z^{2}-\cdots-a_{n}{ }^{n} z_{n}=0,
$$

whose roots are the reciprocals of the roots of (1), and let $\xi$ denote a root of least absolute value of this equation. Write

$$
\frac{1}{1-a_{1} z-a_{2}{ }^{2} z^{2}-\cdots-a_{n}{ }^{n} z^{n}}=1+c_{1} z+c_{2} z^{2}+\cdots .
$$

Now, the circle of convergence of the power series in the second member of this equation passes through the point $\xi$. Its radius of convergence is therefore equal to $|\xi|=1 / X$. Therefore by a well-known property of power series we have

$$
X=\limsup _{m=\infty} \sqrt[m]{\left|c_{m}\right|} .
$$

But from (10) it is clear that we have

$$
\begin{aligned}
1+\left(a_{1} z+a_{2}{ }^{2} z^{2}+\cdots+a_{n}{ }^{n} z^{n}\right)+\left(a_{1} z\right. & \left.+\cdots+a_{n}{ }^{n} z^{n}\right)^{2}+\cdots \\
& =1+c_{1} z+c_{2} z^{2}+\cdots .
\end{aligned}
$$

Expanding the parenthesis quantities in the first member by means of the multinomial formula and equating coefficients of like powers of $z$, we have

$$
c_{m}=\Sigma \frac{\left(t_{1}+t_{2}+\cdots+t_{n}\right) !}{t_{1} ! t_{2} ! \cdots t_{n} !} a_{1}^{t_{1}} a_{2}{ }^{2 t_{2}} a_{3}{ }^{3 t_{3}} \cdots a_{n}{ }^{n t_{n}},
$$

where the summation extends over all the non-negative values $t_{1}, t_{2}, \cdots, t_{n}$ for which $t_{1}+2 t_{2}+3 t_{3}+\cdots+n t_{n}=m$. Taking (11) and (12) together we have the desired explicit formula for $X$ in terms of the coefficients of equation (1).

Though the value of $X$ given in (11) and (12) is exact it is far too unwieldy to be useful in applications.

By comparing the second member of (12) with the multinomial expansion of $\left(\left|a_{1}\right|+\left|a_{2}\right|+\cdots+\left|a_{n}\right|\right)^{m}$ and making 
use of (11) one may readily show that

$$
X \leqq\left|a_{1}\right|+\left|a_{2}\right|+\cdots+\left|a_{n}\right| .
$$

(A more elementary proof of this formula is given in $\S 7$ below.) It is obvious that the upper bound in (13) is actually attained in the case of binomial equations.

5. Employing the method of Fujiwara,* we start with the inequality

$$
\rho^{n} \leqq\left|a_{1}\right| \rho^{n-1}+\left|a_{2}\right|^{2} \rho^{n-2}+\cdots+\left|a_{n}\right|^{n},
$$

which must obviously be satisfied by the absolute value $\rho$ of any root of equation (1). If we suppose that

$$
\rho^{n}>\lambda_{k}\left|a_{k}\right|^{k} \rho^{n-k} \quad(k=1,2, \cdots, n),
$$

while the positive quantities $\lambda$ have the sum of their reciprocals not greater than unity, we shall evidently be led to a contradiction with (14). Hence we have the central theorem of Fujiwara's paper, namely, $\dagger$

$$
X \leqq \max \left\{\lambda_{k}^{1 / k}\left|a_{k}\right|\right\} \quad(k=1,2, \cdots, n),
$$

provided that the positive quantities $\lambda$ satisfy the relation

$$
\frac{1}{\lambda_{1}}+\frac{1}{\lambda_{2}}+\cdots+\frac{1}{\lambda_{n}} \leqq 1 \text {. }
$$

Let us consider the question as to what values of the $\lambda$ 's will make the bound afforded by (15) the lowest possible. It is easy to see that they are the values which render equal the $n$ quantities in the second member of (15) and are such that the equality sign in (16) affords a valid relation. Supposing the $\lambda$ 's chosen in this way, let us write

$$
\sigma=\lambda_{k}^{1 / k}\left|a_{k}\right| \quad(k=1,2, \cdots, n) .
$$

Solving these equations for $\lambda_{k}$ and substituting in (16) (with the sign of inequality replaced by the equality sign), we have

$$
\sigma^{n}=\left|a_{1}\right| \sigma^{n-1}+\left|a_{2}\right|^{2} \sigma^{n-2}+\cdots+\left|a_{n}\right|^{n} .
$$

It is easy to see that this equation has a single positive root. Hence we are led to the following theorem, which we state in the notation of the third form of equation (1):

\footnotetext{
* Tôhoku Math. Journal, vol. 10 (1916), pp. 167-171.

$\dagger$ The theorem obviously remains true if the equality sign is expunged from each inequality in it.
} 
No root of the equation

$$
x^{n}=\beta_{1} x^{n-1}+\beta_{2} x^{n-2}+\cdots+\beta_{n}
$$

is in absolute value greater than the positive root of the equation

$$
\sigma^{n}=\left|\beta_{1}\right| \sigma^{n-1}+\left|\beta_{2}\right| \sigma^{n-2}+\cdots+\left|\beta_{n}\right| .
$$

It is evident that this bound is actually attained in the case of any equation in which the $\beta$ 's are positive or zero. Moreover, it is clear that this is the lowest bound that can be obtained by the direct use of equation (14). Furthermore, it is obvious that a close approximation to this bound is readily obtained in the case of any numerical equation.

6. From (16) it follows that $\lambda_{k} \geqq 1$; and thence, when (16) is satisfied, we see that

$$
X \leqq \max \left\{\lambda_{k}{ }^{l_{k}}\left|a_{k}\right|\right\} \quad(k=1,2, \cdots, n),
$$

where $l_{k}$ is any real quantity not less than $1 / k$.

Applying this to an equation of $t+1$ terms, we have the following result:

The maximum absolute value $X$ of a root of the equation

$$
x^{n}=\gamma_{1}^{n-n_{1}} x^{n_{1}}+\gamma_{2}{ }^{n-n_{2}} x^{n_{2}}+\cdots+\gamma_{t-1}{ }^{n-n_{t-1}} x^{n_{t-1}}+\gamma_{t}{ }^{n},
$$

where $n, n_{1}, \cdots, n_{t-1}$ form a decreasing sequence of positive integers, satisfies the relation

$$
X \leqq \max \left\{\lambda_{k}{ }^{1 / k}\left|\gamma_{k}\right|\right\} \quad(k=1,2, \cdots, t),
$$

provided that the positive quantities $\lambda$ are subject to the condition

$$
\frac{1}{\lambda_{1}}+\frac{1}{\lambda_{2}}+\cdots+\frac{1}{\lambda_{t}} \leqq 1 .
$$

If we consider the question as to what values of the $\lambda$ 's will make the bound afforded by (19) the lowest possible we shall be led by the method of $\S 5$ to the following theorem (giving such lowest bound):

No root of equation (18) is in absolute value greater than the positive root of the equation

$$
s^{t}=\left|\gamma_{1}\right| s^{t-1}+\left|\gamma_{2}\right|^{2} s^{t-2}+\left|\gamma_{3}\right|^{3} s^{t-3}+\cdots+\left|\gamma_{t}\right|^{t} .
$$

Taking $\lambda_{k}=t$ in (19), we see that we have for equation 
(18) the relation

$$
X \leqq \max \left\{t^{1 / k}\left|\gamma_{k}\right|\right\} \quad(k=1,2, \cdots, t),
$$

a result given by Cauchy for the case $t=n$.

Next, let us set

$$
\lambda_{k}=\frac{1}{c_{t k}(\sqrt[t]{2}-1)^{k}} .
$$

Then (20) is a valid relation. Hence we have through (19) the inequality

$$
X \leqq \max \left\{\frac{\left|\gamma_{k}\right|}{c_{t k}(\sqrt[t]{2}-1)}\right\} \quad(k=1,2, \cdots, t) .
$$

The special case $t=n$ is the Birkhoff-Jensen upper bound given in (2). The method of proof is that employed for this special case by Fujiwara in the Tôhoku Mathematical Journal, volume 10. For an equation of high degree and relatively few terms the bound is much closer than that of Birkhoff and Jensen.

7. In relation (17) we may choose special values of the quantities $l_{k}$ so as to obtain the relation

$$
\begin{array}{r}
X \leqq \operatorname{Max}\left\{\lambda_{1}\left|a_{1}\right|, \lambda_{2}\left|a_{2}\right|, \cdots, \lambda_{k_{1}}\left|a_{k_{1}}\right|, \lambda_{k_{1}+1^{3}}\left|a_{k_{1}+1}\right|, \cdots,\right. \\
\left.\lambda_{k_{2}}{ }^{\frac{1}{2}}\left|a_{k_{2}}\right|, \lambda_{k_{2}+1^{3}}{ }^{\frac{1}{3}}\left|a_{k_{2}+1}\right|, \cdots, \lambda_{n}{ }^{1 / t}\left|a_{n}\right|\right\},
\end{array}
$$

provided that the positive quantities $\lambda$ satisfy (16). If we consider the question as to what values of the $\lambda$ 's will make the bound afforded by this relation the lowest possible we shall be led by the method of $\S 5$ to the following theorem (giving such lowest bound):

No root of equation (1) is in absolute value greater than the positive root of the equation

$$
\begin{aligned}
r^{t}= & \left(\left|a_{1}\right|+\cdots+\left|a_{k_{1}}\right|\right) r^{t-1}+\left(\left|a_{k_{1}+1}\right|^{2}+\cdots\right. \\
& \left.+\left|a_{k_{2}}\right|^{2}\right) r^{t-2}+\cdots+\left(\left|a_{k_{t-1}+1}\right|^{t}+\cdots+\left|a_{n}\right|^{t}\right) .
\end{aligned}
$$

It is clear that one may prove in a similar way that

No root of equation (1) is in absolute value greater than the positive root of the equation

$$
\begin{aligned}
r^{n}= & \left(\left|a_{1}\right|+\cdots+\left|a_{k_{1}}\right|\right) r^{n-1}+\left(\left|a_{k_{1}+1}\right|^{k_{1}+1}+\cdots\right. \\
& \left.+\left|a_{k_{2}}\right|^{k_{1}+1}\right) r^{n-k_{1}-1}+\cdots+\left(\left|a_{k_{t-1}+1}\right|^{k_{t-1}+1}+\cdots\right. \\
& \left.+\left|a_{n}\right|^{k_{t-1}+1}\right) r^{n-k_{t-1}-1} .
\end{aligned}
$$


In the former of the last two results, take $t=1$; then we have

$$
X \leqq\left|a_{1}\right|+\left|a_{2}\right|+\cdots+\left|a_{n}\right|
$$

a relation obtained in $\S 4$ above by less elementary means.

In (21) let $t=2$ and write

$$
\left|a_{1}\right|+\cdots+\left|a_{k_{1}}\right|=s_{1}, \quad\left|a_{k_{1}+1}\right|^{2}+\cdots+\left|a_{n}\right|^{2}=s_{2} .
$$

Then we see that

$$
X \leqq \frac{1}{2}\left\{s_{1}+\sqrt{s_{1}^{2}+4 s_{2}}\right\} .
$$

In particular, if $a_{1}=0$ we may take $k_{1}=1$ and show that

$$
X \leqq \sqrt{\left|a_{2}\right|^{2}+\left|a_{3}\right|^{2}+\cdots+\left|a_{n}\right|^{2}} .
$$

More generally, if $a_{1}=0=a_{2}=\cdots=a_{\mu-1}$, we have readily from (22) the relation

$$
X \leqq \sqrt[\mu]{\left|a_{\mu}\right|^{\mu}+\left|a_{\mu+1}\right|^{\mu}+\cdots+\left|a_{n}\right|^{\mu}} .
$$

We may also apply to (21) and (22) the theorems already proved for equation (1) and thus obtain other results for equation (1) itself. Thus through use of (15) and (22) we see that

$$
\begin{array}{r}
X \leqq \max \left\{\lambda_{1}\left(\left|a_{1}\right|+\cdots+\left|a_{k_{1}}\right|\right),\left[\lambda _ { 2 } \left(\left|a_{k_{1}+1}\right|^{k_{1}+1}+\cdots\right.\right.\right. \\
\left.\left.\left.+\left|a_{k_{2}}\right|^{k_{1}+1}\right)\right]^{1 /\left(k_{1}+1\right)}, \cdots\right\}
\end{array}
$$

provided that the positive quantities $\lambda$ satisfy relation (20).

8. In (19) let us take

$$
\lambda_{k}=\frac{1}{\left|\gamma_{k}\right|^{k}}\left(\left|\gamma_{1}\right|+\left|\gamma_{2}\right|^{2}+\cdots+\left|\gamma_{t}\right|^{t}\right)
$$

Then (20) is satisfied and from (19) we see that for equation (18) we have

$$
X \leqq \max \left\{\left|\gamma_{1}\right|+\left|\gamma_{2}\right|^{2}+\cdots+\left|\gamma_{t}\right|^{t}\right\}^{1 / k} \quad(k=1,2, \cdots, t) \text {. }
$$

Hence for equation (18) we have

$$
\begin{aligned}
& X \leqq\left\{\sum_{i=1}^{t}\left|\gamma_{i}\right|^{i}\right\}^{1 / t} \text { if } \sum_{i=1}^{t}\left|\gamma_{i}\right|^{i} \leqq 1 ; \\
& X \leqq \sum_{i=1}^{t}\left|\gamma_{i}\right|^{i} \quad \text { if } \sum_{i=1}^{t}\left|\gamma_{i}\right|^{i} \geqq 1 .
\end{aligned}
$$

The method of proof here employed is identical with that 
used by Fujiwara (loc. cit.) for the special case $t=n$. From the first relation in (23) we see readily that

since

$$
X \leqq\left\{t \sum_{i=1}^{t}\left|\gamma_{i}\right|^{2 i}\right\}^{1 / 2 t} \quad \text { if } \quad \sum_{i=1}^{t}\left|\gamma_{i}\right|^{i} \leqq 1
$$

$$
\left(\sum_{i=1}^{t}\left|\gamma_{\imath}\right|^{i}\right)^{2} \leqq t \sum_{i=1}^{t}\left|\gamma_{i}\right|^{2 i}
$$

as one may readily prove.

9. The theorem of Fujiwara stated in relation (15) will also yield the upper bound given in (3). For the third form of equation (1) relation (15) may be written

$$
X \leqq \max \left\{\lambda_{k}\left|\beta_{k}\right|\right\}^{1 / k} \quad\left(k_{i}=1,2, \cdots, n\right),
$$

the positive quantities $\lambda$ being again subject to relation (16). For the proof of (3) it is obviously sufficient to show that (16) is satisfied if the $\lambda$ 's have the values

$$
\lambda_{k}=\frac{1}{\left|\beta_{k}\right| \nu^{k}}, \text { where } \frac{1}{\nu}=\left\{1+\left|\beta_{1}\right|^{2}+\cdots+\left|\beta_{n}\right|^{2}\right\}^{1 / 2} .
$$

For the moment denote the first member of (16) by $S$. Then we have

$$
\begin{aligned}
S & =\left|\beta_{1}\right| \nu+\left|\beta_{2}\right| \nu^{2}+\cdots+\left|\beta_{n}\right| \nu^{n} \\
& \leqq\left\{\left|\beta_{1}\right|^{2}+\cdots+\left|\beta_{n}\right|^{2}\right\}^{1 / 2}\left\{\nu^{2}+\nu^{4}+\cdots+\nu^{2 n}\right\}^{1 / 2} \\
& \leqq\left\{\frac{1}{\nu^{2}}-1\right\}^{1 / 2}\left\{\nu^{2}+\nu^{4}+\cdots+\nu^{2 n}\right\}^{1 / 2} \\
& \leqq\left\{1-\nu^{2 n}\right\}^{1 / 2}<1, \text { since } \nu<1 .
\end{aligned}
$$

Hence (16) is satisfied and (3) is proved.

Since (3) is proved by aid of (15) it is clear that it can never afford a lower bound for $X$ than that given in the italicized theorem of $\S 5$.

In a similar way one may prove the generalization (7) due to Kuniyeda. The method is essentially that of Kuniyeda's memoir. It is easy to see further that (7) can never afford a lower bound for $X$ than that given in the italicized theorem in $\S 5$. 
10. From the third form of equation (1) we have $|x|^{n-k}\left|x^{k}-\beta_{1} x^{k-1}-\cdots-\beta_{k}\right| \leqq\left|\beta_{k+1}\right| \cdot|x|^{n-k+1}+$

Hence if $\cdots+\left|\beta_{n}\right|$.

we have

$$
\left|x^{k}-\beta_{1} x^{k-1}-\cdots-\beta_{k}\right| \geqq r \quad(r>0),
$$

$$
|x|^{n-k} \leqq \frac{1}{r}\left|\beta_{k+1}\right| \cdot|x|^{n-k-1}+\cdots+\frac{1}{r}\left|\beta_{n}\right| .
$$

Therefore the last inequality will certainly be satisfied if

$$
|x|^{k} \geqq\left|\beta_{1}\right| \cdot|x|^{k-1}+\cdots+\left|\beta_{k-1}\right| \cdot|x|+\left(\left|\beta_{k}\right|+r\right) .
$$

Thence it is easy to see that

The greatest absolute value $X$ of a root of equation (1) satisfies the relation

$$
X \leqq \max \left\{\rho_{1}, \rho_{2}\right\},
$$

where $\rho_{1}$ and $\rho_{2}$ are the positive roots of the equations

$$
\begin{gathered}
\rho^{k}=\left|\beta_{1}\right| \rho^{k-1}+\cdots+\left|\beta_{k-1}\right| \rho+\left(\left|\beta_{k}\right|+r\right), \\
\rho^{n-k}=\frac{1}{r}\left|\beta_{k+1}\right| \rho^{n-k-1}+\cdots+\frac{1}{r}\left|\beta_{n}\right|,
\end{gathered}
$$

respectively, $r$ being any positive quantity and $k$ any integer less than $n$.

This theorem itself may obviously be applied to either or both of equations (24) and (25); so that an upper bound to $X$ may be obtained in terms of the solutions of equations of as low degree as one wishes. Thus, as a special case, we may take the equations to be linear and thus obtain the following result (found by Kojima* in a less elementary way):

$$
\begin{aligned}
X \leqq \max \left\{\left|\beta_{1}\right|+r_{1}, \frac{\left|\beta_{k}\right|}{r_{1} r_{2} \cdots r_{k-1}}+r_{k}\right. & \\
& \left.(k=2, \cdots, n-1), \frac{\left|\beta_{n}\right|}{r_{1} r_{2} \cdots r_{n-1}}\right\},
\end{aligned}
$$

where $r_{1}, r_{2}, \cdots, r_{n-1}$ are any positive quantities.

* Tôhoku Math. Journal, vol. 11 (1917), pp. 119-127. The first two special cases noted had been given earlier by the same author, ibid., vol. 5 (1914), pp. 54-60. 
Taking each $r$ equal to unity, we have the interesting special case

$$
X \leqq \max \left\{1+\left|\beta_{1}\right|, 1+\left|\beta_{2}\right|, \cdots, 1+\left|\beta_{n-1}\right|,\left|\beta_{n}\right|\right\} .
$$

Again, it is easy to show also that

$$
X \leqq \max \{|\alpha|+|\beta|,|\beta|+|\gamma|, \cdots,|\lambda|+|\mu|,|\mu|\},
$$

where the quantities $\alpha, \beta, \cdots, \mu$ are defined by the relations

$$
\alpha=\beta_{1}, \beta^{2}=\beta_{2}, \beta \gamma^{2}=\beta_{3}, \beta \gamma \delta^{2}=\beta_{4}, \cdots, \beta \gamma \cdots \lambda \mu^{2}=\beta_{n} .
$$

Through other special choices of the quantities $r$ numerous rather elegant special inequalities may be obtained, several of which are given explicitly by Kojima (loc. cit.).

In case some of the coefficients of equation (1) are zero it may be preferable to employ the second italicized theorem in $\S 6$ and apply to it the principal theorem of this section (or the special cases of the latter).

It is clear that other general formulas may be found for upper bounds to $X$ by employing a sequence of equations each of which is linear or quadratic, such sequence arising by the repeated application of the theorem of this section.

UNIVERSITY OF ILLINOIS, October, 1917.

\section{THE SOLUTION OF THE WAVE EQUATION BY MEANS OF DEFINITE INTEGRALS.}

BY PROFESSOR H. BATEMAN.

ThE wave equation

$$
\frac{\partial^{2} V}{\partial x^{2}}+\frac{\partial^{2} V}{\partial y^{2}}+\frac{\partial^{2} V}{\partial z^{2}}=\frac{1}{c^{2}} \frac{\partial^{2} V}{\partial t^{2}}
$$

is the oldest member of the family of partial differential equations, and although he was born without the second and third terms he soon acquired these and played a prominent part in mathematical physics at a time when very few partial differential equations had become famous. With the advent of the mathematical theory of elasticity and Maxwell's electromagnetic theory of light he gained a new lease of life and more 